\title{
EEDBC-M: Enhancement of Leach-Mobile protocol with Energy Efficient Density-based Clustering for Mobile Sensor Networks (MSNs)
}

\author{
R.U.Anitha \\ Department of MCA \\ Muthayammal Engineering College, \\ Rasipuram-637408 \\ Tamil Nadu \\ India
}

\author{
P.Kamalakkannan,Ph.D \\ PG\& Research Department of Computer Science \\ Government Arts College \\ Salem- 636007 \\ Tamil Nadu \\ India
}

\begin{abstract}
In recent years, Mobile sensor networks (MSNs) are significantly developed in various areas of applications. The most important feature of MSN is that the sensor nodes are small size, limited processing, low power and can able to change their location. In mobility, life time of the sensor nodes is the most critical parameter. Designing the energy efficient and reliable routing protocols for these applications is a great challenge task due to topological change of the networks and resource limitation in the environment. Existing energy efficient cluster based routing protocols like LEACHM, LEACH-ME, and CBR-Mobile are designed for mobile sensor network which is efficient transmission of sensor nodes. But these protocols are not concentrating the wellorganized cluster formation. So there is increase the end-toend data transmission delay and more energy consumption. Therefore this paper propose an Energy Efficient Density Based Clustering for Mobile node (EEDBC-Mobile) algorithm which is on the density based clustering where as DBSCAN algorithm is used for cluster formation that are significantly more effective in discovering clusters of arbitrary shape. Then cluster head selection process is according to a node residual energy, Mobility and density such as node closeness to its neighbours. It also achieves quite uniform cluster head allocation across the network. A careful selection of the cluster head selection parameters can balance load among cluster heads. The Experimental results demonstrate that EEDBC-Mobile algorithm outperforms LEACH-Mobile in terms of the Energy consumption, Network life time, Throughput, Delay and Data Delivery ratio.
\end{abstract}

\section{Keywords}

Mobile Sensor Network, Mobility, Energy efficient, k-density, Network lifetime, Mobility.

\section{INTRODUCTION}

Mobile Wireless Sensor Networks consist of a large number of tiny mobile sensors nodes that are randomly deployed in a significant area to sense the event. These mobile sensor nodes are communicated with each other to form a sensor network. It can able to send the sensed data into data collection point called the sink or base station. Mobile WSNs could become increasingly useful in a variety of potential civil and military applications, such as intrusion detection, habitat and other environmental monitoring, disaster recovery, hazard and structural monitoring, traffic control, inventory management in factory environments and health related applications, etc [1].

Energy efficiency is one of the most important problem in such resource constrained environment, therefore designing energy efficient routing protocols considerably extend lifetime of sensor network. Wireless sensor networks may consist of hundreds or even thousands of sensor nodes, so designing such large size network requires scalable architecture.

Routing in wireless sensor networks is very challenging task due to their unique characteristics, dynamic nature, Architecture and design issues and resource constraints. There are various routing protocols proposed in literature but hierarchical or cluster based protocols are energy efficient, scalable and tends to pro long network lifetime; they are summarized in [3].

The data gathered from the mobile nodes should minimize the overall energy consumption and maximizing the amount of data received to the base station requires an energy efficient method [2]. Cluster-based architecture is an efficient approach for this data transmission. Clustering is a key technique used to extend the lifetime of a sensor network by reducing energy consumption [4][5]. A generic cluster -based WSN architecture is presented in Figure 1.

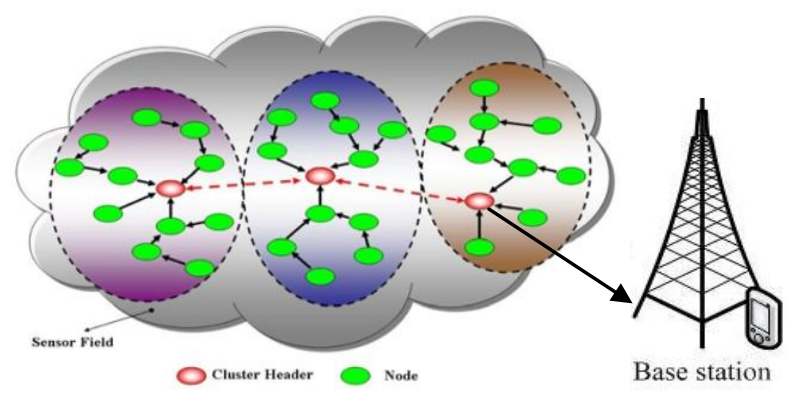

Fig 1.Cluster-based wireless sensor network architecture

In most cluster-based algorithms, a predefined number of clusters and cluster heads are required. LEACH [7] has motivated the design of several other protocols which try to improve the cluster-head selection process. LEACH-Mobile [8] is provoked the mobility behaviour of the sensor nodes. 
In this paper propose an efficient density based clustering algorithms, which are designed to discover the clusters in an arbitrary shape in networks with noise, a cluster is defined as a high-density region partitioned by low-density regions in a space. DBSCAN [12] (Density Based Spatial Clustering of Applications with Noise) is a typical Density-based clustering algorithm which is used to form a clusters in an organized manner. Cluster head selection scheme is done in the basis of remaining energy of the node and density of the node.

The rest of the paper is organized as follows. Section 2 provides an overview of previous work on LEACH protocol in wireless sensor networks. Section 3 briefly describes the problem statement of the existing LEACH-Mobile protocol. Section 4 presents the proposed an algorithm of improved LEACH-Mobile called EEDBC-Mobile (Energy Efficient Density Based Clustering Protocol for mobile Wireless Sensor Networks). Section 5 describes that the simulation environment and presents the simulation model and the parameters used in the experiments. Finally, Section 6 Shows the Simulation Results and Section 7 concludes by a recount of the obtained results. Section 8 provides suggestions for future work.

\section{RELATED WORKS}

This section briefly outlines the related works taken in the area of mobile sensor networks (MSN) which is LEACH [7] protocol improvements.

In LEACH [7], the nodes are organized themselves into local clusters. Each node has the same initial energy because of homogeneous networks. The operation is divided into rounds. In the set-up phase, the $\mathrm{CH}$ is selected from the organized clusters if a random number between 0 and 1 chosen by $\mathrm{CH}$ is less than threshold value. In the steady-state phase each non $\mathrm{CH}$ node sends data and the time slot allocated to $\mathrm{CH}$.The $\mathrm{CH}$ aggregates the data and sends it to the BS. But the cluster formation is initiated in each round is not energy efficient and also it does not support mobility.

As a result, the work done by Do-Seong Kim and Yeong-Jee Chung propose LEACH-Mobile [8] (LEACH-M) routing protocol which applicable to Wireless mobile networks. The basic idea in LEACH-Mobile is to declare the membership of cluster as they move and to confirm whether a mobile sensor node is able to communicate with a specific cluster head. It transmits a message back to mobile sensor node from cluster head for data transmission within a timeslot allocated in TDMA schedule of a wireless sensor cluster. If the mobile sensor node does not receive the data transmission from cluster head within an allocated timeslot according to TDMA schedule, it sending join-request message at next TDMA time slot allocated. Then it decides the cluster to which it will belong for this moment by receiving cluster join-ack messages back from specific cluster heads. But in this protocol, transmission overhead is increased to send a message because of membership declaration.

To move forward this problem, Santhosh Kumar G et al. propose LEACH-Mobile Enhanced [9][10] (LEACH-ME), the node with a lowest mobility factor is selected as a $\mathrm{CH}$. Mobility factor is calculated based on the number of time that a node changes from one cluster to another. In steady state phase, if the non- $\mathrm{CH}$ node may not receive any request packet from $\mathrm{CH}$ node due to mobility then the $\mathrm{CH}$ node does not receive any acknowledgement form the non- $\mathrm{CH}$ nodes. If it is happened two consecutive timeslots, then $\mathrm{CH}$ assume that the node has moved and it deletes the timeslot of the node. However, LEACH-ME consumes much energy for determining mobility factor of the each node.

The effort taken by Samer A.B Awwad et al proposes cluster based Routing protocol for mobile Nodes in WSN [11] [12] (CBR Mobile-WSN) to avoid the packet loss. The idea of this method is that, one of the cluster heads must be free to receive the packet from lost node that cannot receive data request message. The sensor node does not wait for two consecutive failure frames from cluster head to make decision but directly decided that the node has moved out of the cluster after one frame. Thus the data loss is reduced by sending its data to the new free cluster head and sends join acknowledgement message to the cluster heads.

The work done by Lutful Karim and Nidal Nasser propose Fault Tolerant Clustering Protocol for Mobile WSN [13] (FTCP-MWSN) that is not only energy efficient but also reliable. It does not require any extra timeslot for calculating the mobility of sensor node. So that it provide faster data delivery to BS. In the steady phase, $\mathrm{CH}$ assigns timeslots to the member nodes using TDMA scheme. If a node moves into a new cluster then it sends a Join-Request message to $\mathrm{CH}$. Then the $\mathrm{CH}$ does not allocate the node a timeslot until any timeslot becomes free for moving a node out of this cluster.

Cluster-based Energy-efficient Scheme [14] (CES) for Mobile Wireless Sensor Networks (MWSNs) which relies on weighing k-density, residual energy and mobility parameters for cluster-head election. The CES scheme carries out a periodical cluster-head election process after each round. Moreover, CES enables the creation of balanced 2-hop clusters whose size ranges between two thresholds called upper and lower thresholds.

\section{PROBLEM STATEMENT}

The sensor nodes in a Mobile sensor networks (MSNs) are resource constrained particularly with limited energy for the reason that it fixed in a remote area, So it can not able to refill the battery. Designing the energy efficient and reliable routing protocols for these applications is a great challenge due to topological change of the networks and resource limitation in the environment. In order to conserve energy and increase network lifetime, the node should minimize the energy dissipation and optimize communication. It is possible through efficient routing schemes. Mobility is another major issue, as they may contain hundreds and thousands of sensor nodes are in mobile state. Both of the above issues have been addressed efficiently by cluster-based architectures and algorithms particularly LEACH-Mobile. There are few areas in LEACH-Mobile that can be enhanced to compose it more energy efficient and Mobility.

LEACH-Mobile uses the same set-up procedure used in the basic LEACH protocol. It is not concentrating the wellorganized cluster formation. Instead of, the nodes are organized themselves into local clusters. Therefore, there is a possibility that the elected $\mathrm{CHs}$ will be concentrated in one part of the network. So there is increase the end-to-end data transmission delay and more energy consumption. In the cluster head selection phase, routing table is formed after each round of the $\mathrm{CH}$ selection. Hence it also conserve significant amount of energy. However, LEACH-Mobile assumes that the $\mathrm{CHs}$ are stationary. It is not efficient in terms of energy consumptions and data delivery rate because a large number of packets are lost if the $\mathrm{CH}$ keeps moving before selecting a new $\mathrm{CH}$. Transmission overhead is increasing for cluster head 
due to the long range of transmission to the base station and $\mathrm{CH}$ nodes drained their energy quickly, due to additional processing responsibilities like data aggregation.

In addition, the base station rotates randomly for each round and collects the energy level of the nodes in the cluster for cluster head selection. But in mobility centric environment, the cluster head elections based on simply the energy level, without consider the node mobility can produce severe problem. A node with sufficiently rich energy level, taking over the duty of cluster head possessing high mobility, may move out of the cluster, causing the cluster to become headless. The situation causes the cluster to go for a new cluster head. But again the mobility of the nodes is not considered causing the same process to repeat.

\section{PROPOSED ALGORITHM}

In this section, the proposed algorithm is described in detail. First, it defines the cluster formation based on the DBSCAN algorithm and the cluster head selection is done on the basis of the parameters like Minimum Energy consumption, mobility and density. This paper is also explained the design and pseudo-code for the proposed protocol.

\subsection{EEDBC-Mobile Routing Phases}

The EEDBC-Mobile algorithm is mainly consists of two major phases i.e., Set-up phase and Steady-state phase. Set-up phase is the initial phase and this is the phase where all cluster formation and Cluster head selection will takes place. The basic ideas of this phase is DBSCAN[15] clustering algorithm which is used to form a cluster and the election of specific cluster head which is on the basis of residual energy, Mobility and density of the node. Set-up phase is relatively short when compared to the steady state phase.

In the steady-state phase, the cluster head and non-cluster head nodes receive a particular message at a given time slot according to TDMA time schedule of sensor cluster and reorganize the cluster with minimum energy consumption and node mobility. The steady state phase does the actual data transfer between the sensing node and the sink.

\subsection{Set-up phase}

\subsubsection{Cluster Formation}

Clustering is the process of classifying the nodes into different groups by partitioning sets of data into a series of subsets called clusters. Clusters can be separate as a high density regions or low density region. Density here is considered as the number of nodes in the "neighborhood". Clustering is a technique to achieve high data density. Density Based clustering is an efficient clustering method that determines the adequate number of clusters as well as provides the appropriate position for any cluster to initiate[21].

In this paper, Density based Spatial Clustering of Applications with Noise (DBSCAN) [15] algorithm is used for cluster formation. It is a density based algorithm which discovers clusters with arbitrary shape. The input parameters required for this algorithm is, Eps- radius of the cluster and MinPts minimum nodes required inside the cluster. The basic idea behind this DBSCAN [15] algorithm is as follows,

Eps - neighborhood of a Node: The Eps neighborhood of a node $p$, denoted by NEps ( $p$ ) is defined by
$\operatorname{NEps}(p)=\{p \in D B \mid \operatorname{dist}(p, q) \leq$ Eps $\}$

There are two kinds of nodes in the cluster, the node which is inside the cluster are called core nodes, and nodes on the border of the cluster are called border nodes which shows in Figure 2.

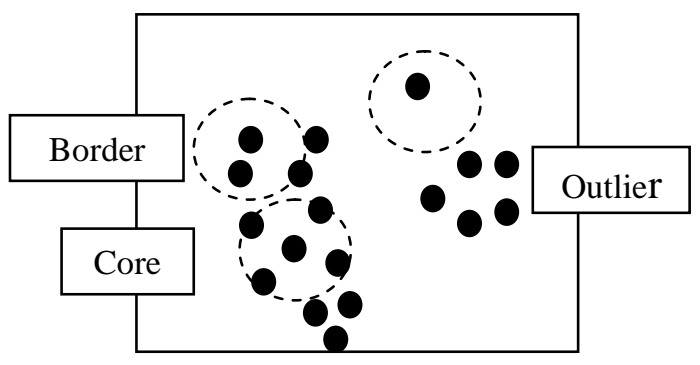

Figure 2: Core and Border nodes in Clusters formation

Directly density-reachable: A node $p$ is directly densityreachable from a node q wrt. Eps, MinPts if

1) $\mathrm{p} \in \operatorname{NEps}(\mathrm{q})$ and

2) $|\operatorname{NEps}(\mathrm{q})| \geq \square$ MinPts

Density-reachable: A node $p$ is density-reachable from a node $\mathrm{q}$ wrt. Eps and MinPts if there is a chain of nodes $\mathrm{p} 1 \ldots$ $\mathrm{pn}, \mathrm{p} 1=\mathrm{q}, \mathrm{pn}=\mathrm{p}$ such that $\mathrm{pi}+1$ is directly density-reachable from pi as shown in Figure 3.

Density-connected: A node $p$ is density-connected to a node $q$ wrt. Eps and MinPts if there is a node o such that both $p$ and $\mathrm{q}$ are density-reachable from o wrt. Eps and MinPts as shown in Figure 4.

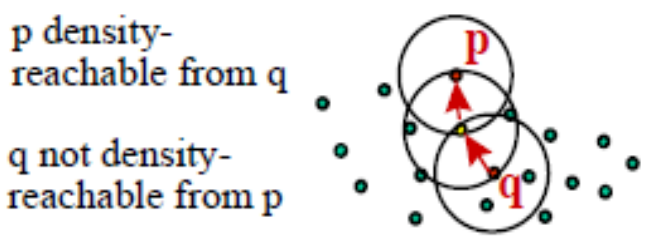

Figure 3: Density-reachable in DBSCAN

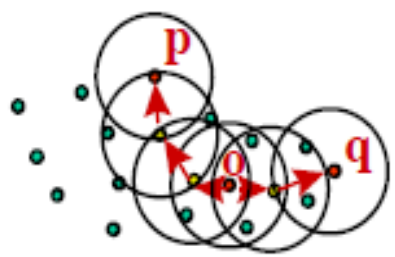

$\mathrm{p}$ and $\mathrm{q}$ densityconnected to each other by $o$

Figure 4: Density-connectivity in DBSCAN

Cluster: Let D be a database of nodes. A Cluster C wrt. Eps and MinPts is a non-empty subset of D satisfying the following conditions:

1) $\forall p$, q: if $p \in C$ and $q$ is density-reachable from $p$ wrt. Eps and MinPts, then q $\in$ C. (Maximality)

2) $\square \forall \mathrm{p}, \mathrm{q} \in \mathrm{C}: \mathrm{p}$ is density-connected to q wrt. Eps and MinPts (Connectivity)

Noise: The noise node is defined as the set of points in the database DB not belonging to any cluster $C$, i.e. noise $=\{p \in$ $\mathrm{DB} \mid \forall: \mathrm{p} \notin \mathrm{C}\}$. 
During the Cluster Formation phase, the Density based Spatial Clustering of Applications with Noise (DBSCAN) algorithm starts with an arbitrary selects a starting node $p$ that has not been visited and it retrieves all neighbor nodes density reachable from starting node $\mathrm{p}$ with respect to Eps and MinPts. If the number of neighbors is greater than or equal to MinPts then the cluster is formed. And also it finds the Core nodes and Border nodes. Neither Core nodes nor Border node in the region will be marked as Noise node.

The Core node initiates a clustering algorithm and nearby nodes will be added into the queue for the further expansion. Each nodes in a queue will be popped out and find the Eps neighbor node for the popped out node. When the new node is a core node, all its neighbor nodes will be assigned with the current cluster id. Then its unprocessed neighbor nodes will be pushed into queue for further processing. This process will be repeated until there are no nodes in the queue for the further processing.

DBSCAN could merge two clusters into one cluster, if two clusters of different density are "close" to each other. Let the distance between two sets of nodes $\mathrm{C} 1$ and $\mathrm{C} 2$ be defined as $\operatorname{dist}(\mathrm{C} 1, \mathrm{C} 2)=\min \{\operatorname{dist}(\mathrm{p}, \mathrm{q}) \mid \mathrm{p} \in \mathrm{C} 1, \mathrm{q} \in \mathrm{C} 2\}$. Consequently, a recursive call of DBSCAN may be necessary for the detected clusters with a higher value for MinPts. The following illustrate the pseudo code of DBSCAN.

\section{Pseudo-code of the Proposed EDBC-Mobile Protocol:}

Input: DB - database of nodes, Eps - neighborhood radius, MinPts - minimum nodes required inside the cluster.

\section{Output: Set of clusters}

\section{CLUSTER FORMATION}

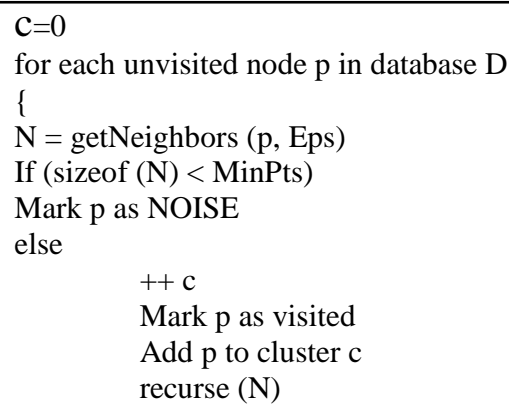

\section{CLUSTER NODE CALCULATION}

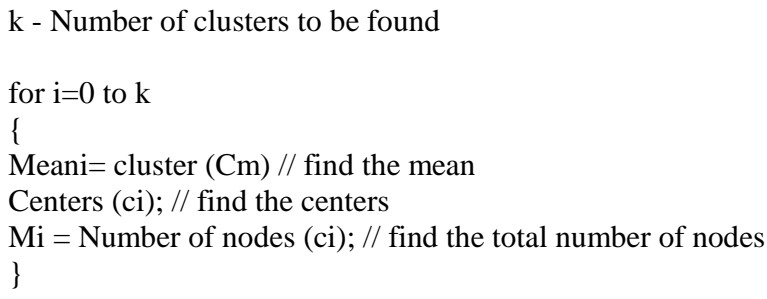

\section{CLUSTER EXPANSLON}

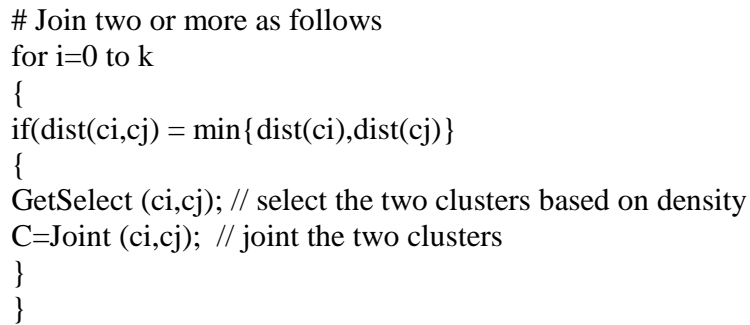

\subsubsection{Cluster Heads Selection}

In the following section Cluster head selection is done based on the algorithm considers the nodes residual energy and nodes density as the parameters. The entire network is divided into more than number of clusters based on the Density based Spatial Clustering of Applications with Noise (DBSCAN) algorithm.

The primary parameter of residual energy of the node is calculated with the help of first order radio model. The mobile sensor nodes can evaluate themselves to find the remaining energy of their nodes within the cluster. If some nodes are having the residual energy is less than minimum energy then those nodes are deleted from the network. Then the secondary parameter of node density that is the number of cluster members in each cluster can calculate within the clusters. The computed density value and remaining energy must be broadcasts as locally to all its neighbors. From this the clusters themselves select the highest residual energy and highest density node as a cluster head for that cluster. By receiving this value, each node is able to know which node will be the cluster head.

After the cluster head has been determined the each cluster head broadcasts an advertisement message to all other nodes using a CSMA MAC protocol and inviting the other nodes to join in the cluster. Other nodes may also participate in the advertising process. Hearing these advertisement messages, each sensor node chooses the nearest cluster head and registers itself as a cluster member. 
$\mathrm{r} \leftarrow \operatorname{random}(0,1)$;

if $(\operatorname{Einit}(\mathrm{s})>0 \& \mathrm{r} \bmod (1 /$ popt $) \neq 0)$

\{ compute $\mathrm{T}(\mathrm{s})$; //given by (1)

for $\mathrm{i}=0$ to $\mathrm{k}$

\{ $\quad \mathrm{Mj}=$ Number of nodes (ci);

$\mathrm{m}=$ highest $(\mathrm{Mj})$;

if $(\mathrm{r}<\mathrm{T}(\mathrm{s})$ and $\mathrm{Mj}(\mathrm{t})=\mathrm{m})$

\{ $\mathrm{CH}\{\mathrm{s}\}=\mathrm{TRUE} ; / /$ node $\mathrm{s}$ be a $\mathrm{CH}$

else $\{\mathrm{CH}\{\mathrm{s}\}=F A L S E ; / /$ node s not be a $\mathrm{CH}$

\}

if $(\mathrm{CH}\{\mathrm{s}\}=\mathrm{TRUE})$

\{ $\quad \mathrm{BC}(\mathrm{ADV}) \leftarrow$ broadcast an advertisement message Join(IDi); // non-CH node $\mathrm{i}$ join into the closest $\mathrm{CH}$

Cluster(c); //form a cluster c; \}

\subsection{Steady State Phase}

After all the sensed data has been received from the non- $\mathrm{CH}$ nodes, the $\mathrm{CH}$ can make a data aggregation function. In this function, $\mathrm{CH}$ should be removing the redundant data and compresses the data into a single packet. This packet is transmitted into the base station through multi hops transmission. Each cluster member will know its respective time slot. If the time slot is allotted then only the sensed data transmitted to the Cluster head otherwise it goes to sleep.

In addition, it transmits the value of its remaining power. The cluster-head maintains a table which records the node with maximum power at current round. After it has forwarded the data to the sink, it selects the node with maximum remaining power as the cluster-head for the next round. The pseudo code for steady state phase is explained as follows.

\section{DATA TRANSMISSION}

\section{If $(\mathrm{CH}(\mathrm{s})=$ TRUE $)$ \\ \{ \\ Receive(IDi, DataPCK) //receive data from} members;

Aggregate(IDi, DataPCK) //aggregate received data; TransToBS(IDi, DataPCK); //transmit received

data;

else If (MyTimeSlot=TRUE)

\{

TransToCH(IDi, DataPCK); //transmit sensed data;

\}

else

\{

SleepMode $(i)=T R U E ; / /$ node $i$ at a sleep state
As state earlier, DBSCAN algorithm is used to form a cluster in the network. After the certain period of time, the network follows the same procedure formation to compose a reclusters, as described earlier. The computation of threshold value should depend upon the application. Once re-clustering is completed, Base Station constantly observes the residual energy and Mobility of the existing $\mathrm{CH}$. If it is below the threshold value then it select another $\mathrm{CH}$ based on same conditions, described earlier. Finally the $\mathrm{CH}$ should be checked out the routing path. If the routing path residual energy goes below the threshold or any node fails, BS selects another path and sends the routing path to the respective $\mathrm{CH}$.

\section{SIMULATION ENVIRONMENT}

In this section, simulations are used to analyse and evaluate the performance of the algorithm. This paper uses the network simulator called NS2 [14], which developed by UC Berkeley to simulate the method. To verify the improved proposed algorithm, the results are compared with LEACH-Mobile algorithm.

\subsection{Simulation Platform}

In this section a comparative study between the behaviours of the routing protocols. LEACH-Mobile and EDBC-Mobile will be given by simulation of MWSN chosen to represent application. The well known NS2 [17] simulation tool is used. It is a discrete event network simulator for networking research. NS2 provides a complete development environment for performance evaluation of communication networks and distributed systems.

It provides a substantial support to simulate group of protocols like TCP, UDP, FTP, HTTP and DSR [18]. The purpose of the study was to investigate the behaviour of LEACH-Mobile and EDBC-Mobile for Energy consumption, Packet Delivery Ratio, Data Reliability and Latency are the parameters.

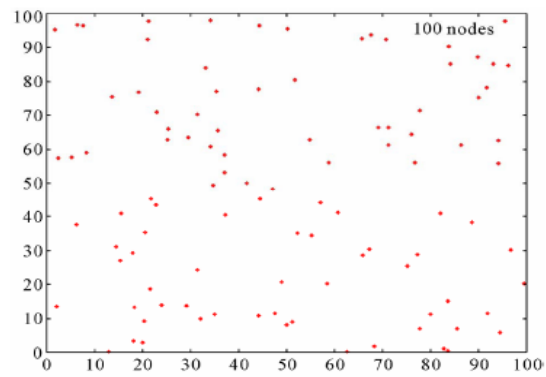

Figure 5: Random deployment of 100 Mobile sensor nodes in the field.

\subsection{Simulation Setup}

As shown in Figure 5, 100 mobile sensor nodes were arranged randomly in the field $100 \mathrm{~m} \times 100 \mathrm{~m}$ in the sensor fields. The size of each packet was set to 512 Bytes. The time for transmitting such a packet is considered as 1 unit of delay. Simulation time for each scenario was set to 500 seconds and repetitive simulations for each scenario were performed to verify the reliability of the results.

The energy consumption due to communication will be calculated using the first order energy model. The sink nodes were also arranged. The nodes have mobile in nature, and they can correspond with the sink nodes directly. The initial energy

\subsection{Re-clustering and Re-routing Phase}


of a sensor node is 2 Joule and the energy of a sink node is infinite. The settings of other parameters of the network environment were displayed in Table I.

TABLE 1.Simulation Parameters and their values

\begin{tabular}{|c|c|}
\hline Parameter & Value \\
\hline Number of Mobile nodes & 100 \\
\hline Network size & $100 \mathrm{~m}$ x 100m \\
\hline Initial Energy & 2 Joule \\
\hline Data Packet size & 512 Bytes \\
\hline Nodes Movement & Mobility \\
\hline Simulation time & 600 seconds \\
\hline $\begin{array}{c}\text { Energy consumption for sending data } \\
\text { packets }\end{array}$ & 50 pJoule \\
\hline Energy consumption in free space/air & 0.01 pJoule \\
\hline Mobility Model & Random Way Point \\
\hline Communication Model & Bi-directional \\
\hline
\end{tabular}

\subsection{Selected Performance Metrics for Evaluation}

In order to compare the EEDBC-Mobile and LEACH-Mobile protocol's scalability, the quantitative metrics are used to measure and evaluate the performance of the simulation. A set of performance metrics are used for comparing the proposed protocol of this work is shown in Figure 6. Each of these

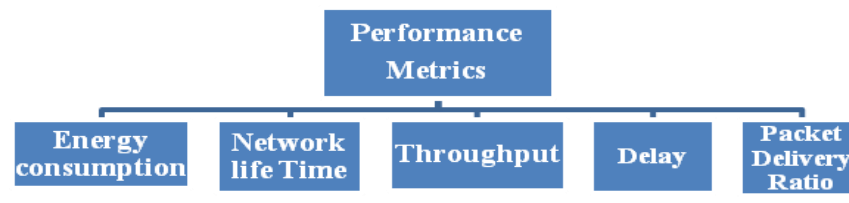

metrics parameters can be described briefly as follows [16] [19].

Figure 6: A set of Performance Metrics

\subsubsection{Average Energy Consumption (Ea):}

The average energy consumption is calculated across the entire topology. It measures the average difference between the initial level of energy and the final level of energy that is left in each node. Let $\mathrm{Ei}=$ the initial energy level of a node, $\mathrm{Ef}$ $=$ the final energy level of a node and $\mathrm{N}=$ number of nodes in the simulation. The Average energy consumption is calculated from the following Eq. (3)

$$
E_{\mathrm{a}}=\frac{\sum_{\mathrm{k}=\mathrm{i}}^{\mathrm{n}}\left(\mathrm{E}_{\mathrm{ik}}-\mathrm{E}_{\mathrm{fk}}\right)}{\mathrm{N}}
$$

This metric is an important because the energy level of the network uses is proportional to the network's lifetime. The lower the energy consumption the longer is the network's lifespan.

\subsubsection{Network life time}

It is a time interval from starting operation of the sensor network until the death of the first alive node. Network lifetime is the time span from the deployment to the instant when the network is considered nonfunctional. It can be, for example, the instant when the first sensor dies, a percentage of sensors die, the network partitions, or the loss of coverage occurs. The average Network life time of the Network is measured as the following Eq. (4)

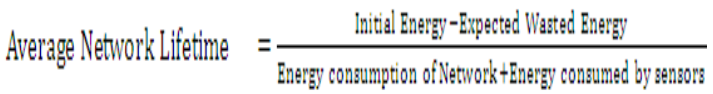

\subsubsection{Throughput:}

The ratio of total data received by a receiver from a sender for a time the last packet received by receiver measures in $\mathrm{bit} / \mathrm{sec}$ and byte/sec. It can be expressed mathematically as the Eq. (5)

$$
\text { Throughput (bit/sec) }=\frac{\text { Number of Delivered Packat:Packet sizes8 }}{\text { Total duration of aimulation }}
$$

\subsubsection{Average End-to-End Delay of Data Packets}

It is the average delay between the sending of the data packet by the source and its receipt at the corresponding receiver including the delays due to route acquisition, buffering and processing at intermediate nodes, and retransmission delays at the MAC layer, etc. if the value of End-to-end delay is high then it means the protocol performance is not good due to the network congestion. The Average Delay can be calculated form the following Eq. (6)

$$
\begin{aligned}
& \text { Average Delay }=\frac{\sum_{i=0}^{\mathrm{n}} \text { Time Packet Received }- \text { Time Packat zenti }}{\text { Total Number of Packets Received }} \\
& \text { 5.3.5 Packet Delivery Ratio }
\end{aligned}
$$

This represents the ratio between the number of data packets that are sent by the source and the number of data packets that are received by the sink. The Average packet Delivery Ratio is calculated from the following Eq. (7)

$$
\text { Packet Delivery Ratio }=\frac{\text { Successfully Delivered Packets }}{\text { Reqired Packets }}
$$

This metric indicates both the loss ratio and the effort required to receive data of the routing protocol. If both the data are equal then the ratio should be equal to 1 which is called as ideal ratio. If the ratio falls below the ideal ratio, then it could be an indication of some faults in the protocol. Conversely, if the ratio is higher than the ideal ratio, then it is an indication that the sink receives a more than number data packet which is called as redundant packets. It is not advantageous because receiving repeated packets will consume more energy [21].

\section{EXPERIMENTAL RESULTS}

In this section presents the performance of the proposed algorithm EEDBC-Mobile obtained by simulation using NS2 [13].Performance evaluation and comparison between LEACH-Mobile and EDBC-Mobile are shown in the following Figure 7, 8, 9, 10 and 11 where mobile sensor nodes were evenly distributed within a $100 \mathrm{~m} \times 100 \mathrm{~m}$ area.

In figure 7, the number of mobile nodes is used as $\mathrm{X}$ axis and Average energy consumption as $\mathrm{Y}$ axis. From this graph, it can be observed that the Average energy consumption of EEDBC-Mobile algorithm is less than LEACH-Mobile 
Enhanced, LEACH-Mobile and LEACH as shown in the Figure 7. This provides confirmation that the proposed EEDBC-Mobile protocol improves the life time of the MWSN as compare to LEACH-ME and LEACH-M.

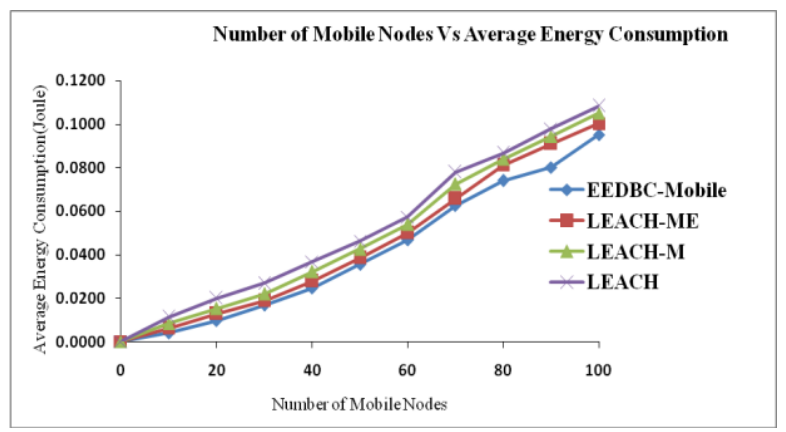

Figure 7: Average energy per packet under different number of sensor nodes

Figure 8 depicts the network life time. It can be observed that EEDBC-Mobile showed better results with respect to the network lifetime. In EEDBC-Mobile, since the energy consumption is more evenly distributed the number of nodes that shut down decreases.

Figure 9 shows the comparisons of throughput obtained using EEDBC-Mobile, LEACH-Mobile Enhanced, LEACH-Mobile and LEACH protocols. Results show that EEDBC-Mobile as a trust protocol similar to LEACH-ME and LEACH-ME, but LEACH provides fewer throughputs when compared to other protocols.

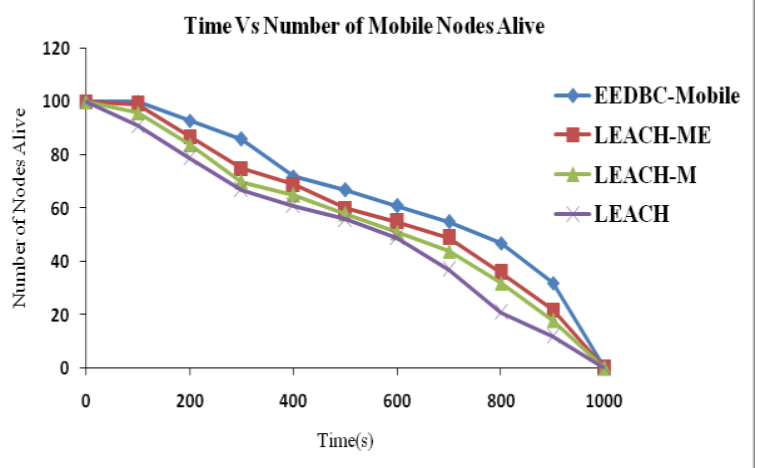

Figure 8: Network Life Time under different number of sensor nodes

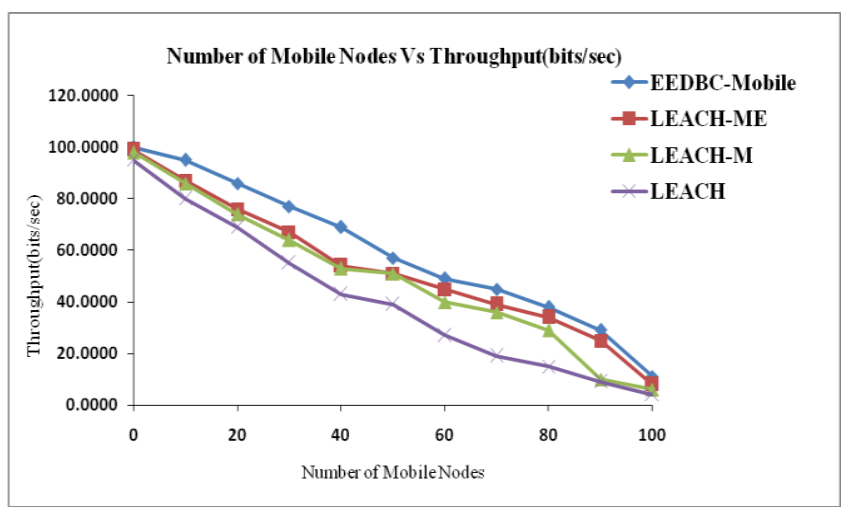

Figure 9: Throughput under different number of sensor nodes
Figure 10 presents the delay taken by the routing protocols during the simulation period 50 and 100 number of frames for all scenarios. It is clear from figures that LEACH gains the highest delay, especially on large size of nodes. LEACH-M exhibits large packet delay because its routes discovery takes more time. LEACH-ME gives the lowest delay as compared to LEACH-M. But EEDBC-Mobile gives very low delay as compared to other protocols.

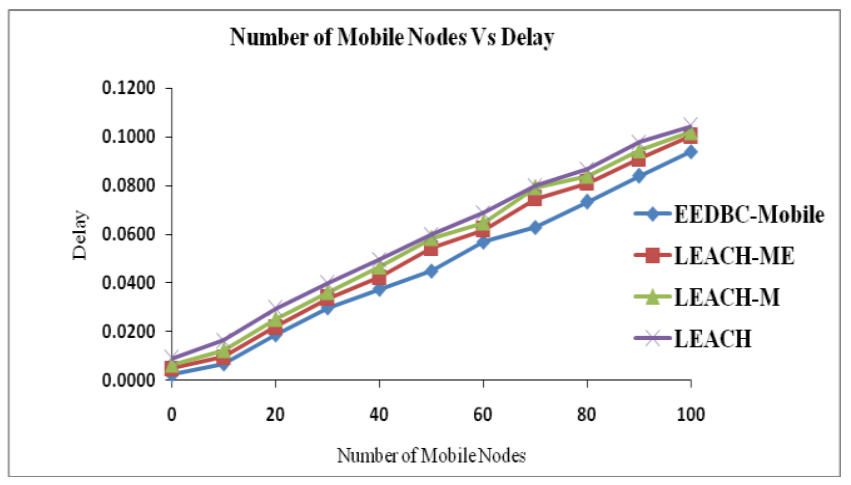

Figure 10: Delay under different number of sensor nodes

Figure 11 proves that the good degree of reliability while transmit as a multiple paths are kept in the routing table. The routing process in LEACH-Mobile and EEDBC-Mobile is similar to LEACH [7]. It is clear from the figure 9 shows that packet delivery ratio of EEDBC-Mobile achieved more when compared to LEACH-ME, LEACH-M and LEACH protocols.

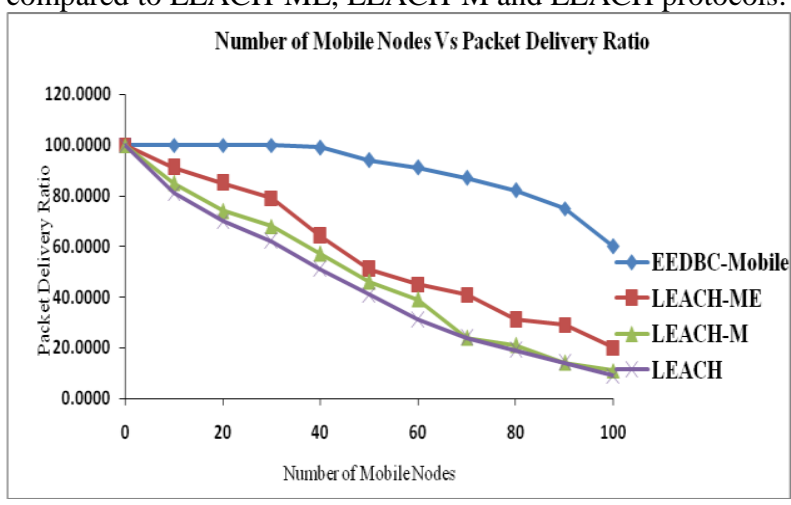

Figure 11: Packet Delivery Ratio under different number of sensor nodes

\section{CONCLUSION}

This paper has presented EEDBC-Mobile an enhancement of the LEACH-Mobile protocol. The proposed protocol uses a technique that provides an optimal clustering technique with a use of DBSCAN algorithm to better organized and wellformed clusters. The cluster heads are nominated by considering the residual energy, mobility and density of the mobile sensor nodes. The cluster-head keeps a table which records the node with maximum power at current round. It is reduced the utilization of the energy during the round time of a sensor network communication protocol. This protocol is judge against with energy efficient protocols like LEACHMobile and LEACH-Mobile Enhanced. The simulation results shows that EEDBC-Mobile is much better when compared to LEACH-Mobile in terms of different metrics like Energy consumption, Network life time, Throughput, delay and data delivery ratio. 


\section{FUTURE WORK}

In future work, it would be plan to do a broad simulations for the proposed protocol that mobility of the node would be taken as a cluster head selection and attach the gateway nodes as an intermediate node to transmit the data to the base station. This paper presents part of the continuing work being conducted to consider round-time optimization and other techniques to achieve fair allocation of cluster head energy dissipation.

\section{REFERENCE}

[1] C. Y. Chong, S. P. Kumar, "Sensor networks: Evolution, opportunities, and challenges", Proc IEEE, vol. 91 No 8, pp. 1247-1256, 2003.

[2] V. Rodoplu, T. H. Meng, "Minimum Energy Mobile Wireless Networks", IEEE Journal on selected areas in communications, vol. 17, no. 8, August 1999, pp-13331344.

[3] K. Akkaya, M. F. Younis: A survey on routing protocols for wireless sensor networks. Elsevier Ad Hoc Network Journal, 3(3):325-349, 2005.

[4] Hania Aoudia, Youcef Touati, Arab Ali-Cherif and Patrick Greussay "Hierarchical Routing Approach-Based Energy Optimization in Wireless Sensor Networks" MobiWac'12, October 21-22, 2012, Paphos, Cyprus.Copyright ACM 978-1-4503-1623-1/12/10.

[5] Hania Aoudia, Youcef Touati, Arab Ali-Cherif and Patrick Greussay "Hierarchical Routing Approach-Based Energy Optimization in Wireless Sensor Networks" MobiWac'12, October 21-22, 2012, Paphos, Cyprus.Copyright 2012 ACM 978-1-4503-1623$1 / 12 / 10 \ldots \$ 15.00$.

[6] Mai Thi Quynh Banh, Giang Trung Nguyen,Thu Quynh Ngo "Energy balanced and fault-tolerant clustering routing protocol for event driven mwsn",soICT 2012, August 23-24 ,2012,Ha-Long ,Vietnam Copyright 2012 ACM 978-1-4503-1232-5

[7] Wendi B.Heinzelman,Anantha P.Chandrakasan and Hari Balakrishnan "An Application-Specific Protocol Architecture for Wireless Microsensor networks",IEEE Transactions on wireless communications vol.1 NO.4 2002.

[8] Do-Seong Kim and Yeong-Jee Chung, "SelfOrganization Routing Protocol Supporting Mo bile Nodes for Wireless Sensor Network", Proceedings of the First International Multi-Symposiums on Computer and Computational Sciences (IMSCCS'06), Volume 02, IEEE Computer Society Washington, DC, USA (C2006 ISBN:0-7695-2581-4, Pages 622-626.

[9] Kumar, G.S.; Vinu Paul, M.V.; Jacob, K.P. "Mobility Metric based LEACH-Mobile Protocol", Advanced Computing and Communications, 2008. ADCOM 2008. 16th International Conference on, On page(s): $248-253$
[10] Kumar, G.S.; Vinu Paul, M.V.; Athithan, G.; Jacob, K.P. "Routing protocol enhancement for handling node mobility in wireless sensor networks", TENCON 2008 2008 IEEE Region 10 Conference, On page(s): 1 - 6.

[11] Samer A. Awwad, Chee Kyun Ng , Nor K. Noordin , Mohd. Fadlee Rasid, Cluster Based Routing Protocol for Mobile Nodes in Wireless Sensor Network, Wireless Personal Communications: An International Journal, v.61 n.2, p.251-281, November 2011.

[12] S. A. B. Awwad, C. K. Ng, N. K. Noordin and M. F. A. Rasid, "Cluster based routing protocol for mobile nodes in wireless sensor network," in Collaborative Technologies and Systems, IEEE Transaction on wireless communications 2009, pp. 233-241.

[13] Karim, L.; Nasser, N. "Energy Efficient and Fault Tolerant Routing Protocol for Mobile Sensor Network", Communications (ICC), 2011 IEEE International Conference on, On page(s):1-5.

[14] Lehsaini M., Guyennet H. and Feham, M., "CES: Cluster-based Energy-efficient Scheme for Mobile Wireless Sensor Networks "2008, in IFIP International Federation for Information Processing, Volume 264; Wireless Sensor and Actor Networks II; Ali Miri; (Boston: Springer), pp. 13-24.

[15] Jiawei Han, Micheline Kamber, "Data Mining: Concepts and Techniques", Morgan Kaufmann Publishers, 2002.

[16] F. Ye, G. Zhong, S. Lu, and L. Zhang, "GRAdient broadcast: a robust data delivery protocol for large scale sensor networks," ACM Transaction on Wireless Networks, vol. 11, no. 3, pp. 285-298, 2005.

[17] Network Simulator, http://www.isi.edu/nsnam/ns.

[18] http://www.isi.edu/nsnam/ns/ns-documentation.html.

[19] R.U.Anitha and Dr.P.Kamalakkannan, "A Survey on Energy Efficient Routing Protocols in Wireless Sensor Networks", International Research Journal of Mobile and Wireless Communications, Vol 03, Issue 01, ISSN: 2249-6491 pp 100-105, January-April 2012.

[20] R.U.Anitha and Dr.P.Kamalakkannan,"Energy Efficient Cluster Head Selection Algorithm in Mobile Wireless Sensor Networks “,In the Proceedings of International Conference on Computer Communication and Informatics (ICCCI -2013) Sri Sakthi Institute of Engineering and Technology, Coimbatore $04-06$ January,2013 (C) 2013 IEEE Explore (Indexed by Scopus).

[21] R.U.Anitha and Dr.P.Kamalakkannan," Improvement on Leach-Mobile by Efficient Density-Based Clustering for Mobile Nodes in WSN",In the Proceedings of International Conference on Innovations in Communication, information and Computing (ICICIC2013) Jan 09-11 at Sasurie College of Engineering pp.612,2013. 


\section{Authors Biography}

Prof. R.U.Anitha was born in Tamilnadu, India in 1981. She received the B.Sc, MCA and M.Phil degrees from Periyar University, Salem and 2001, 2004, and 2007 respectively. From 2004 she has been teaching to the student community. She is working towards her Ph.D degree at the Computer Applications department of Anna University of Technology, Coimbatore. Her research interest includes Wireless sensor networks and Wireless Adhoc Networks.
Dr.P.Kamalakkannan was born in Tamilnadu, India in 1967. He received his B.Sc, and MCA degrees from Bharathiar University, Coimbatore and Ph.D degree from Periyar University, Salem. Since 1991 he has been teaching to the student community as Professor, Head and Director. He is guiding $12 \mathrm{PhDs}$. His area of research includes Computer Networks, Mobile Communication, Image Processing, Parallel and Distributed Computing. He is having more than 50 National and International research Journals and conference publications. $\mathrm{He}$ is a Journal Reviewer of IJOPCM, IJCSA and also a reviewer of Various International conferences. 